\title{
INTERNAÇÕES HOSPITALARES POR CONDIÇÕES SENSÍVEIS À ATENÇÃO PRIMÁRIA À SAÚDE
}

\author{
Hospital admissions for sensitive conditions to primary health care \\ Ingresos hospitalarios por condiciones sensibles de la atención primaria de salud
}

\author{
Karina Gama dos Santos Sales iD \\ Escola Superior de Ciências da Santa Casa de Misericórdia de Vitória - EMESCAM - Vitória (ES) - Brasil \\ Luiz Carlos de Abreu (iD \\ Escola Superior de Ciências da Santa Casa de Misericórdia de Vitória - EMESCAM - Vitória (ES) - Brasil \\ José Lucas Souza Ramos iD \\ Escola Superior de Ciências da Santa Casa de Misericórdia de Vitória - EMESCAM - Vitória (ES) - Brasil \\ Italla Maria Pinheiro Bezerra iD \\ Escola Superior de Ciências da Santa Casa de Misericórdia de Vitória - EMESCAM - Vitória (ES) - Brasil
}

\section{RESUMO}

Objetivo: Analisar as internações hospitalares por condições sensíveis à Atenção Primária à Saúde (APS) no estado de Minas Gerais. Métodos: Estudo ecológico com delineamento de série temporal, de base populacional, realizado no estado de Minas Gerais, acerca da internação hospitalar e as principais causas de internações por condições sensíveis à APS. A análise foi baseada na Autorização de Internação Hospitalar, considerando custos, ano, sexo e faixa etária, de acordo com os dados do Sistema de Informação Hospitalar (SIH). Os microdados foram extraídos do serviço de transferência de arquivos no Departamento de Informática do Sistema Único de Saúde (DATASUS) em junho de 2018. Os registros analisados são referentes ao período de janeiro de 2008 a dezembro de 2017, sendo incluídas todas as internações por condições sensíveis à Atenção Primária à Saúde. Resultados: Evidenciou-se que, no período de 2008 a 2017, as internações sensíveis à atenção primária representaram $21,21 \%$ (2.506.782) do total de internações no estado de Minas Gerais, com a região Jequitinhonha, seguida das regiões Nordeste e CentroSul, contribuindo com os maiores percentuais. Com relação às causas, a maior prevalência se concentra em doenças do aparelho circulatório 54,22\% (816.617), seguida pelas doenças do aparelho respiratório 43,48\% (586.809), doenças endócrinas 85,70\% (325.082), doenças geniturinárias 37,13\% (309.281) e doenças infecciosas e parasitárias 38,37\% (266,294). Conclusão: As internações hospitalares por condições sensíveis à Atenção Primária à Saúde no estado de Minas Gerais seguem o padrão nacional. Embora as internações estejam decrescendo, ainda representam mais de $20 \%$ do total de internações realizadas.

Descritores: Atenção Primária à Saúde; Hospitalização; Assistência à Saúde.

\section{ABSTRACT}

Objective: To analyze hospital admissions for conditions sensitive to Primary Health Care in the state of Minas Gerais, Brazil. Methods: It is an ecological study with population-based time-series design in the state of Minas Gerais, concerning the hospital admissions and the main causes of them for conditions sensitive to primary care. The analysis was performed based on the Hospitalization Authorization according to the costs, year, gender, and age group based on data from the Hospital Information System (SIH). The micro data were extracted from the file transfer service at the Department of Informatics of the Unified Health System (DATASUS) in June 2018. The records analyzed refer to the period from January 2008 to December 2017, including all hospitalizations for conditions sensitive to primary health care. Results: It was evident that from 2008 to 2017, hospitalizations sensitive to primary care accounted for $21.21 \%(2,506,782)$ of the total hospitalizations in the state of Minas Gerais, with Jequitinhonha Region followed by the Northeast and Center South contributing to the higher percentages. Regarding causes, the highest prevalence is concentrated in circulatory system diseases $54.22 \%(816,617)$, followed by respiratory system diseases $43.48 \%(586,809)$, endocrine diseases $85.70 \%(325,082)$, genitourinary diseases $37,13 \%(309,281)$ and infectious and parasitic diseases $38.37 \%(266,294)$. Conclusion: hospital admissions due to conditions sensitive to Primary Health Care in the state of Minas Gerais follow a national pattern, but although hospitalizations are decreasing, it still represents more than $20 \%$ of all hospitalizations.

Descriptors: Primary Health Care; Hospitalization; Delivery of Health Care. 


\section{RESUMEN}

Objetivo: Analizar los ingresos hospitalarios por condiciones sensibles de la Atención Primaria de Salud (APS) del estado de Minas Gerais. Métodos: Estudio ecológico de delineamiento de serie temporal, de base poblacional realizado en el estado de Minas Gerais sobre el ingreso hospitalario y las principales causas de ingreso por condiciones sensibles de la APS. El análisis está basado en el Permiso de Ingreso Hospitalario considerando los costes, el año, el sexo y la franja de edad, según los datos del Sistema de Información Hospitalario (SIH). Se han identificado los micros datos del servicio de transferencia de archivos del Departamento Informático del Sistema Único de Salud (DATASUS) en junio de 2018. Los registros analizados son del período entre enero de 2008 y diciembre de 2017 y se han incluido todos los ingresos por condiciones sensibles de la Atención Primaria de Salud. Resultados: Se ha evidenciado que en el período entre 2008 y 2017 los ingresos sensibles de la atención primaria han representado el 21,21\% (2.506.782) del total de ingresos del estado de Minas Gerais, con la región de Jequitinhonha, seguida de las regiones Noreste y CentroSur contribuyendo con los mayores porcentajes. Respecto las causas, la mayor prevalencia está en las enfermedades del aparato circulatorio con el 54,22\% (816.617), seguido de las enfermedades del aparato respiratorio con el 43,48\% (586.809), las enfermedades endócrinas con el 85,70\% (325.082), las enfermedades genitourinarias con el 37,13\% (309.281) y las enfermedades infecciosas y parasitarias con el 38,37\% (266,294). Conclusión: Los ingresos hospitalarios por condiciones sensibles de la Atención Primaria de Salud del estado de Minas Gerais siguen el patrón nacional. Aunque los ingresos disminuyen aún representan más del $20 \%$ del total de ingresos realizados.

Descriptores: Atención Primaria de Salud; Hospitalización; Prestación de Atención de Salud.

\section{INTRODUÇÃO}

O Sistema Único de Saúde (SUS) está voltado para o atendimento das necessidades de saúde da população, tendo em vista o desenvolvimento de ações e serviços que assegurem os cuidados agudos e/ou agudizados, e aqueles que demandam certa continuidade, que contam, ainda, com ações de prevenção de doenças e promoção da saúde ${ }^{(1)}$.

A Atenção Primaria à Saúde (APS) vem se expandindo no Brasil desde sua criação, justamente pelo fato de possibilitar essa cobertura para a população. Novos programas e estratégias vêm sendo criadas para tentar minimizar os problemas, por isso é importante fazer a inclusão da população no SUS ${ }^{(2,3)}$.

Os aspectos tratados na APS estão em constantes avanços. Afinal, precisam acompanhar as necessidades atuais, principalmente nas questões que visam prevenir doenças que podem levar à morte e promover saúde, especialmente em relação a doenças que podem ser evitáveis quando são realizadas ações capazes de orientar e educar os usuários ${ }^{(2)}$.

A Política Nacional de Atenção Básica (PNAB) ressalta a importância de ofertar e organizar essas ações e serviços da APS para que seja possível garantir melhor e amplamente o acesso integral à saúde. Em 2011, a PNAB induziu revisões de diretrizes para a Atenção Básica de Saúde, destacando oferta de recursos extras para os serviços que criassem novas equipes ${ }^{(2,4)}$.

A avaliação dos indicadores de saúde é importante para apresentar o atual cenário e as ações de saúde, possibilitando novos olhares e melhores planejamentos de saúde, pois direcionam a construção de novos olhares e ações mais qualificadas para atender a necessidade específica de cada região(5).

Esses dados são referências à gestão do SUS, que tem como objetivo melhorar esses indicadores, os níveis de atenção à saúde da população, reduzir os riscos e melhorar a qualidade de vida. Para isso, é importante considerar a diversidade, a mobilidade e os diferentes sujeitos, entre outras características essenciais para a determinação desses indicadores ${ }^{(6)}$.

Entre as morbidades hospitalares, é interessante destacar as internações por condições sensíveis à atenção primária (ICSAP), uma vez que podem ser atendidas oportuna e efetivamente em unidades básicas de saúde, sem necessidade de hospitalização( ${ }^{(7)}$. Entendendo que as ICSAP constituem um evento que pode ser evitado por meio de intervenções preventivas realizadas na atenção primária, diminuindo os agravos ocasionados aos pacientes. Portanto, essas hospitalizações servem para pressupor como estão o acesso, a cobertura, a qualidade e o desempenho da atenção primária, e também o seu papel de ordenadora das redes de atenção à saúde ${ }^{(8)}$

No Brasil, a lista de ICSAP inclui 19 causas de hospitalização e diagnósticos de acordo com a décima revisão da Classificação internacional de doenças e causas de morte (CID-10). Portanto, estudos têm sido desenvolvidos descrevendo o perfil geral de internações ou de prevalência de ICSAP utilizando dados secundários, com o intuito de retratar a capacidade e a resolutividade da Atenção Primária à Saúde (APS) no contexto das redes de atenção à saúde ${ }^{(9)}$. 
Sendo assim, justifica-se a realização do presente estudo frente à reforma da política nacional de atenção básica e a necessidade da avaliação das ações da atenção primária à saúde, buscando a redução de custos em serviços hospitalares por causas sensíveis a esse nível de atenção, além de auxiliar na identificação de possíveis focos de ação para promoção da saúde. Frente a isto, questiona-se como está a ocorrência de internações por condições sensíveis à atenção primária e qual o seu impacto nos custos orçamentários do estado de Minas Gerais, bem como nas suas macrorregiões de saúde. Dessa forma, o presente estudo tem por objetivo analisar as internações hospitalares por condições sensíveis à atenção primária no estado de Minas Gerais.

\section{MÉTODOS}

Trata-se de estudo ecológico, com delineamento de série temporal, com utilização de microdados de natureza secundária referente às internações por condições sensíveis à atenção primária (ICSAP) de residentes em Minas Gerais, Brasil.

O estado de Minas Gerais apresenta população de 19.597.330(6), considerando-se o Plano Diretor de Regionalização da Saúde de Minas Gerais (PDR/MG), é estratificado em 13 regiões ampliadas de saúde, a saber: 3101 Sul; 3102 CentroSul; 3103 Centro; 3104 Jequitinhonha; 3105 Oeste; 3106 Leste; 3107 Sudeste; 3108 Norte; 3109 Noroeste; 3110 Leste do Sul; 3111 Nordeste; 3112 Triângulo do Sul; 3113; Triângulo do Norte ${ }^{(10)}$.

Foram coletados dados sobre morbidade hospitalar de acordo com a Classificação Internacional de Doenças (CID-10), identificando as principais causas de ICSAP, tendo como unidade de análise as internações de residentes no estado de Minas Gerais, Brasil.

Utilizaram-se microdados de natureza secundária referentes às internações hospitalares provenientes do Sistema de Informações Hospitalares do SUS (SIH/SUS), tendo como instrumento básico a Autorização de Internação Hospitalar (AlH), inseridas no período entre 2008 a 2017, no qual se obteve todos os atendimentos provenientes de internações hospitalares financiadas pelo SUS.

Como critério de seleção, consideraram-se todas as internações por local de residência, ocorridas no período entre 2008 e 2017, cuja causa básica fizesse parte de um desses grupos: doenças preveníveis por imunização; gastrenterites infecciosas e complicações; anemia; deficiências nutricionais; infecções de ouvido, nariz e garganta; pneumonias bacterianas; doenças pulmonares; hipertensão; asma; angina; insuficiência cardíaca; doenças cerebrovasculares; diabetes mellitus; epilepsias; infecção no rim e no trato urinário; infecção da pele e tecido subcutâneo; doenças inflamatórias em órgãos pélvicos femininos; úlcera gastrointestinal e doenças relacionadas ao pré-natal e parto ${ }^{(11)}$.

Não foram contabilizadas as internações hospitalares realizadas fora do Sistema Único de Saúde, como hospitalizações privadas custeadas diretamente pelo paciente ou pelo sistema suplementar de saúde (convênios e seguros de saúde), e, portanto, não registradas pelo SIH.

O SIH/SUS registra mais de $85 \%$ das internações de pessoas que procuram hospitais públicos e privados registradas no SUS e inclui $92,3 \%$ de unidades de saúde no Brasil.

Extraíram-se os microdados, em junho de 2018, do serviço transferência de arquivos fornecido pelo DATASUS (website: www.datasus.gov.br). Para consulta dos dados, utilizaram-se os programas TabNet e TabWin. Realizou-se a coleta de dados com dois pesquisadores independentes para identificar possíveis discrepâncias.

Para este estudo, utilizaram-se informações correspondentes à data de internação do paciente na unidade hospitalar por local de residência. Nesse caso, o estado de Minas Gerais. Os dados obtidos representam cem por cento dos casos que foram notificados e alimentados no SIH/SUS de maneira correta.

A análise estatística descritiva foi baseada nas $\mathrm{AlH}$, de acordo com custo, ano, sexo e faixa etária, considerando as causas sensíveis à APS no contexto global das internações hospitalares.

O presente estudo envolve apenas a descrição e análise de dados secundários de população, obtidos pelo recenseamento geral de população, coletados junto ao Sistema de Informações sobre Mortalidade, e de internações hospitalares, informados pelo SIH/SUS. Todas essas fontes de informação são de domínio público, dispensando, assim, a apreciação por comitê de ética em pesquisa com seres humanos. Não há informações adicionais que não fossem de livre acesso e, em especial, nenhuma informação com identificação individual foi obtida para a realização do estudo.

\section{RESULTADOS}

No período de janeiro de 2008 a dezembro de 2017, foram realizadas 11.819 .746 internações nos serviços públicos de saúde no estado de Minas Gerais. Destas, 21,21\% (2.506.782) são internações por condições sensíveis à atenção primária (ICSAP), com uma média de 20 internações por mês. 
O valor financeiro das Internações por condições sensíveis à atenção primária correspondeu a 17,24\% do total de custos com internações, e as que mais demandaram custos foram, também, as doenças do aparelho circulatório $(45,39 \% ; R \$ 1.408 .927 .884,90)$, seguidas pelas doenças do aparelho respiratório $(35,06 \% ; R \$ 504.416 .798,24)$ e doenças endócrinas $(79,83 \% ; R \$ 174.093 .233,99)$. Embora as doenças do aparelho geniturinário tenham apresentado maior número de internações que as doenças infeciosas e parasitárias $(11,94 \%$; $\$$ 132.048.075,35), as doenças infeciosas tiveram um custo maior que aquelas (Tabela I).

Tabela I - Internações hospitalares pelo Sistema Único de Saúde de acordo com os capítulos da CID-10 no estado de Minas Gerais, Brasil, 2008 a 2017.

\begin{tabular}{|c|c|c|c|c|c|}
\hline Capítulo & Morbidades & ICSAP & $\%$ & $\begin{array}{l}\text { Não } \\
\text { ICSAP }\end{array}$ & $\%$ \\
\hline I & Algumas doenças infecciosas e parasitárias & 266.294 & 38.37 & 427.651 & 61,63 \\
\hline II & Neoplasias & - & - & 736.602 & 100,00 \\
\hline III & $\begin{array}{l}\text { Doenças do sangue e dos órgãos hematopoiéticos e alguns } \\
\text { Transtornos imunitários }\end{array}$ & 14.021 & 12,87 & 94.963 & 87,13 \\
\hline IV & Doenças endócrinas, nutricionais e metabólicas & 325.082 & 85,70 & 54.229 & 14,30 \\
\hline $\mathbf{v}$ & Transtornos mentais e comportamentais & - & - & 332.639 & 100,00 \\
\hline VI & Doenças do sistema nervoso & 78.027 & 36,65 & 134.851 & 63,35 \\
\hline VII & Doenças do olho e anexos & - & - & 91.492 & 100,00 \\
\hline VIII & Doenças do ouvido e da apófise mastoide & 2.056 & 13,49 & 13.188 & 86,51 \\
\hline IX & Doenças do aparelho circulatório & 816.617 & 54,22 & 689.623 & 45,78 \\
\hline $\mathbf{x}$ & Doenças do aparelho respiratório & 586.809 & 43,48 & 762.751 & 56,52 \\
\hline $\mathbf{X I}$ & Doenças do aparelho digestivo & 43.054 & 3,91 & 1.058 .458 & 96,09 \\
\hline XII & Doenças da pele e do tecido subcutâneo & 44.228 & 22,14 & 155.571 & 77,86 \\
\hline XIII & Doenças do sistema osteomuscular e do tecido conjuntivo & - & - & 252.561 & 100,00 \\
\hline XIV & Doenças do aparelho geniturinário & 309.281 & 37,13 & 523.690 & 62,87 \\
\hline $\mathbf{x V}$ & Gravidez, parto e puerpério & 21.047 & 0,97 & 2.144 .658 & 99,03 \\
\hline $\mathbf{X V I}$ & Algumas afecções originadas no período perinatal & 266 & 0,11 & 237.185 & 99,89 \\
\hline XVII & $\begin{array}{l}\text { Más-formações congênitas, deformidades e anomalias } \\
\text { cromossômicas }\end{array}$ & - & - & 72.042 & 100,00 \\
\hline XVIII & $\begin{array}{l}\text { Sintomas, sinais e achados anormais de exames clínicos e de } \\
\text { laboratório, não classificados em outra parte }\end{array}$ & - & - & 155.932 & 100,00 \\
\hline $\mathbf{X I X}$ & $\begin{array}{l}\text { Lesões, envenenamentos e algumas outras consequências } \\
\text { de causas externas }\end{array}$ & - & - & 1.144 .384 & 100,00 \\
\hline $\mathbf{x x}$ & Causas externas de morbidade e de mortalidade & - & - & 2.873 & 100,00 \\
\hline $\mathbf{X X I}$ & $\begin{array}{l}\text { Fatores que influenciam o estado de saúde e o contato com } \\
\text { os serviços de saúde }\end{array}$ & - & - & 227.619 & 100,00 \\
\hline \multirow[t]{2}{*}{ XXII } & Códigos para propósitos especiais & - & - & 02 & 100,00 \\
\hline & Total & 2.506 .782 & 21,21 & 9.312 .964 & 78,79 \\
\hline
\end{tabular}

Fonte: Sistema de Informação Hospitalar do Sistema Único de Saúde (SIH/SUS). Dados do Departamento de Informática do Sistema Único de Saúde (DATASUS - www.datasus.gov.br). Ministério da Saúde. Brasil ICSAP: Internações por Condições Sensíveis à Atenção Primária 
Dos pacientes com maior prevalência de ICSAP, 52\% (1.304.059) são mulheres e 46,4\% (1.163.763) com idade igual ou superior a 60 anos (Tabela II).

Tabela II - Valor das internações por condições sensíveis a atenção primária pelo Sistema Único de Saúde de acordo com os capítulos da CID-10 no estado de Minas Gerais, Brasil, 2008 a 2017.

\begin{tabular}{|c|c|c|c|c|c|}
\hline \multirow{2}{*}{ Capítulo } & \multirow{2}{*}{ Morbidades } & \multicolumn{2}{|l|}{ ICSAP } & \multicolumn{2}{|l|}{ Não ICSAP } \\
\hline & & Valor & $\%$ & Valor & $\%$ \\
\hline 1 & Algumas doenças infecciosas e parasitárias & $R \$ 132.048 .075,35$ & 11,94 & $\mathrm{R} \$ 974.102 .661,28$ & 88,06 \\
\hline II & Neoplasias & - & 0,00 & $\mathrm{R} \$ 1.338 .011 .555,34$ & 100,0 \\
\hline III & $\begin{array}{l}\text { Doenças do sangue e dos órgãos hematopoiéticos } \\
\text { e alguns Transtornos imunitários }\end{array}$ & $\mathrm{R} \$ 5.040 .037,99$ & 7,70 & $\mathrm{R} \$ 60.419 .465,50$ & 92,30 \\
\hline IV & Doenças endócrinas, nutricionais e metabólicas & $R \$ 174.093 .233,99$ & 79,83 & $\mathrm{R} \$ 43.973 .356,40$ & 20,17 \\
\hline $\mathbf{v}$ & Transtornos mentais e comportamentais & - & - & $\mathrm{R} \$ 336.802 .926,97$ & 100,0 \\
\hline VI & Doenças do sistema nervoso & $\mathrm{R} \$ 52.203 .412,89$ & 18,76 & $\mathrm{R} \$ 226.118 .375,89$ & 81,24 \\
\hline VII & Doenças do olho e anexos & & 0,00 & $\mathrm{R} \$ 96.554 .876,02$ & 100,0 \\
\hline VIII & Doenças do ouvido e da apófise mastóide & $\mathrm{R} \$ 573.697,08$ & 1,43 & $\mathrm{R} \$ 39.536 .442,62$ & 98,57 \\
\hline IX & Doenças do aparelho circulatório & $\mathrm{R} \$ 1.408 .927 .884,90$ & 45,39 & $\mathrm{R} \$ 1.695 .073 .336,75$ & 54,61 \\
\hline $\mathbf{x}$ & Doenças do aparelho respiratório & $\mathrm{R} \$ 504.416 .798,24$ & 35,06 & $\mathrm{R} \$ 934.200 .979,82$ & 64,94 \\
\hline $\mathbf{X I}$ & Doenças do aparelho digestivo & $\mathrm{R} \$ 38.840 .746,45$ & 3,93 & $\mathrm{R} \$ 949.274 .815,40$ & 96,07 \\
\hline XII & Doenças da pele e do tecido subcutâneo & $\mathrm{R} \$ 24.662 .242,29$ & 17,87 & $\mathrm{R} \$ 113.374 .132,55$ & 82,13 \\
\hline XIII & $\begin{array}{l}\text { Doenças do sistema osteomuscular e do tecido } \\
\text { conjuntivo }\end{array}$ & - & 0,00 & $\mathrm{R} \$ 419.881 .239,90$ & 100,0 \\
\hline XIV & Doenças do aparelho geniturinário & $\mathrm{R} \$ 105.763 .344,25$ & 15,91 & $\mathrm{R} \$ 558.893 .480,53$ & 84,09 \\
\hline xV & Gravidez, parto e puerpério & $\mathrm{R} \$ 3.842 .541,90$ & 0,29 & $\mathrm{R} \$ 1.299 .379 .817,25$ & 99,71 \\
\hline $\mathbf{X V I}$ & Algumas afecções originadas no período perinatal & $\mathrm{R} \$ 277.553,38$ & 0,04 & $\mathrm{R} \$ 774.833 .098,30$ & 99,96 \\
\hline XVII & $\begin{array}{l}\text { Más-formações congênitas, deformidades e } \\
\text { anomalias cromossômicas }\end{array}$ & - & 0,00 & $\mathrm{R} \$ 230.047 .139,95$ & 100,0 \\
\hline XVIII & $\begin{array}{l}\text { Sintomas, sinais e achados anormais de exames } \\
\text { clínicos e de laboratório, não classificados em } \\
\text { outra parte }\end{array}$ & - & 0,00 & $\mathrm{R} \$ 142.062 .458,49$ & 100,0 \\
\hline $\mathbf{X I X}$ & $\begin{array}{l}\text { Lesões, envenenamentos e algumas outras } \\
\text { consequências de causas externas }\end{array}$ & - & 0,00 & $\mathrm{R} \$ 1.412 .470 .245,90$ & 100,0 \\
\hline$x x$ & Causas externas de morbidade e de mortalidade & - & 0,00 & $\mathrm{R} \$ 2.597 .984,52$ & 100,0 \\
\hline $\mathbf{X X I}$ & $\begin{array}{l}\text { Fatores que influenciam o estado de saúde e o } \\
\text { contato com os serviços de saúde }\end{array}$ & - & 0,00 & $\mathrm{R} \$ 118.976 .795,71$ & 100,0 \\
\hline \multirow[t]{2}{*}{ XXII } & Códigos para propósitos especiais & - & 0,00 & $\mathrm{R} \$ 573,92$ & 100,0 \\
\hline & Total & $R \$ 2.450 .689 .568,71$ & 17,24 & $R \$ 11.766 .585 .75,01$ & 82,76 \\
\hline
\end{tabular}

Fonte: Sistema de Informação Hospitalar do Sistema Único de Saúde (SIH/SUS). Dados do Departamento de Informática do Sistema Único de Saúde (DATASUS - www.datasus.gov.br). Ministério da Saúde. Brasil

ICSAP: Internações por Condições Sensíveis à Atenção Primária 
Com relação à procedência dos pacientes internados por Macrorregiões de saúde no estado de Minas Gerais, observou-se a diferença entre os cenários regionais sob a perspectiva das ICSAP, porém não sendo possível a visualização em nível municipal. Os maiores índices de ICSAP foram encontrados nas Macrorregiões Jequitinhonha $(30,32 \% ; 64.318)$ e Nordeste $(30,70 \% ; 165.336)$, ambas apresentando um percentual maior que $30 \%$ de ICSAP em comparação ao total geral de internações no período (Tabela III). Resguardando as devidas proporções, tal percentual é superior ao alcançado por Minas Gerais, que obteve, no mesmo período, 21,21\% (2.506.782) de ICSAP (Tabela IV).

As principais causas dessas internações foram relacionadas às doenças do aparelho circulatório $(54,22 \%$; 816.617), doenças do aparelho respiratório (43,48\%; 586.809), doenças endócrinas (85,70\%; 325.082), doenças geniturinárias $(37,13 \% ; 309.281)$ e doenças infecciosas e parasitárias $(38,37 \% ; 266.294)$, respectivamente, conforme demonstra a Tabela I.

Tabela III - Distribuição das internações pelo sistema público de saúde de residentes nas Macrorregiões de Saúde do estado de Minas Gerais, Brasil, 2008 a 2017.

\begin{tabular}{|c|c|c|c|c|}
\hline Região Ampliada de Saúde & ICSAP & $\%$ & Não ICSAP & $\%$ \\
\hline 3101 Sul & 369.028 & 22,02 & 1.306 .640 & 77,98 \\
\hline 3102 Centro Sul & 121.648 & 25,42 & 356.975 & 74,58 \\
\hline 3103 Centro & 578.003 & 17,24 & 2.774 .875 & 82,76 \\
\hline 3104 Jequitinhonha & 64.318 & 30,32 & 147.832 & 69,68 \\
\hline 3105 Oeste & 134.077 & 20,71 & 513.184 & 79,29 \\
\hline 3106 Leste & 204.807 & 23,32 & 673.483 & 76,68 \\
\hline 3107 Sudeste & 303.131 & 24,56 & 931.062 & 75,44 \\
\hline 3108 Norte & 197.698 & 20,22 & 779.906 & 79,78 \\
\hline 3109 Noroeste & 61.945 & 20,27 & 243.647 & 79,73 \\
\hline 3110 Leste do Sul & 86.869 & 20,77 & 331.304 & 79,23 \\
\hline 3111 Nordeste & 165.336 & 30,07 & 384.550 & 69,93 \\
\hline 3112 Triângulo do Sul & 91.668 & 23,08 & 305.493 & 76,92 \\
\hline 3113 Triângulo do Norte & 128.254 & 18,53 & 564.013 & 81,47 \\
\hline Total & 2.506 .782 & 21,21 & 9.312 .964 & 78,79 \\
\hline
\end{tabular}

Fonte: Sistema de Informação Hospitalar do Sistema Único de Saúde (SIH/SUS). Dados do Departamento de Informática do Sistema Único de Saúde (DATASUS - www.datasus.gov.br). Ministério da Saúde. Brasil ICSAP: Internações por Condições Sensíveis à Atenção Primária 
Tabela IV - Distribuição das cinco causas mais frequentes de internações, por faixa etária, pelo Sistema Único de Saúde, por condições sensíveis a atenção primária, de acordo com os capítulos da CID-10 no estado de Minas Gerais, Brasil, 2008 a 2017.

\begin{tabular}{|c|c|c|c|c|}
\hline Faixa etária & & ICSAP & Total & Taxa (\%) \\
\hline & $1^{a}$ & Doenças pulmonares & 33.848 & 25,38 \\
\hline & $2^{\mathrm{a}}$ & Gastroenterites Infecciosas e complicações & 25.839 & 19,38 \\
\hline \multirow[t]{6}{*}{ Menor de 1 ano } & $3^{\mathrm{a}}$ & Pneumonias bacterianas & 24.238 & 18,18 \\
\hline & $4^{\mathrm{a}}$ & Asma & 16.578 & 12,43 \\
\hline & $5^{\mathrm{a}}$ & Infecção no rim e trato urinário & 11.556 & 8,67 \\
\hline & & Outras causas (ICSAP) & 21.289 & 15,96 \\
\hline & $1^{\mathrm{a}}$ & Gastroenterites Infecciosas e complicações & 104.383 & 33,63 \\
\hline & $2^{\mathrm{a}}$ & Asma & 57.133 & 18,41 \\
\hline \multirow[t]{6}{*}{1 a 13 anos } & $3^{a}$ & Pneumonias bacterianas & 56.272 & 18,13 \\
\hline & $4^{\mathrm{a}}$ & Infecção no rim e trato urinário & 21.156 & 6,82 \\
\hline & $5^{\mathrm{a}}$ & Epilepsias & 17.307 & 5,58 \\
\hline & & Outras causas (ICSAP) & 54.140 & 17,44 \\
\hline & $1^{a}$ & Infecção no rim e trato urinário & 24.826 & 36,82 \\
\hline & $2^{\mathrm{a}}$ & Gastroenterites Infecciosas e complicações & 10.339 & 15,33 \\
\hline \multirow[t]{6}{*}{14 a 19 anos } & $3^{\mathrm{a}}$ & Doença relacionada ao pré-natal e parto & 6.274 & 9,30 \\
\hline & $4^{\mathrm{a}}$ & Pneumonias bacterianas & 4.081 & 6,05 \\
\hline & $5^{a}$ & Diabetes mellitus & 4.011 & 5,95 \\
\hline & & Outras causas (ICSAP) & 17.903 & 26,55 \\
\hline & $1^{\mathrm{a}}$ & Infecção no rim e trato urinário & 81.000 & 28,01 \\
\hline & $2^{\mathrm{a}}$ & Gastroenterites Infecciosas e complicações & 34.170 & 11,82 \\
\hline \multirow[t]{6}{*}{20 a 39 anos } & $3^{a}$ & Doença Inflamatória órgãos pélvicos femininos & 21.944 & 7,59 \\
\hline & $4^{\mathrm{a}}$ & Pneumonias bacterianas & 18.143 & 6,27 \\
\hline & $5^{a}$ & Diabetes melitus & 17.223 & 5,96 \\
\hline & & Outras causas (ICSAP) & 116.701 & 40,36 \\
\hline & $1^{\mathrm{a}}$ & Insuficiência cardíaca & 85.485 & 15,75 \\
\hline & $2^{a}$ & Angina & 66.120 & 12,18 \\
\hline \multirow[t]{6}{*}{40 a 59 anos } & $3^{\mathrm{a}}$ & Doenças cerebrovasculares & 61.028 & 11,25 \\
\hline & $4^{\mathrm{a}}$ & Infecção no rim e trato urinário & 51.842 & 9,55 \\
\hline & $5^{\mathrm{a}}$ & Diabetes mellitus & 50.522 & 9,31 \\
\hline & & Outras causas (ICSAP) & 227.668 & 41,95 \\
\hline & $1^{a}$ & Insuficiência cardíaca & 269.893 & 23,19 \\
\hline & $2^{\mathrm{a}}$ & Doenças cerebrovasculares & 164.102 & 14,10 \\
\hline \multirow[t]{5}{*}{60 anos e mais } & $3^{\mathrm{a}}$ & Doenças pulmonares & 128.251 & 11,02 \\
\hline & $4^{\mathrm{a}}$ & Angina & 99.610 & 8,56 \\
\hline & $5^{\mathrm{a}}$ & Infecção no rim e trato urinário & 86.811 & 7,46 \\
\hline & & Outras causas (ICSAP) & 415.096 & 35,67 \\
\hline & & Total & 2.506.782 & \\
\hline
\end{tabular}

Fonte: Sistema de Informação Hospitalar do Sistema Único de Saúde (SIH/SUS). Dados do Departamento de Informática do Sistema Único de Saúde (DATASUS - www.datasus.gov.br). Ministério da Saúde. Brasil ICSAP: Internações por Condições Sensíveis à Atenção Primária 
Tabela $V$ - Distribuição das causas mais frequentes, por sexo, de internações no Sistema Único de Saúde por condições sensíveis a atenção primária, de acordo com os capítulos da CID-10 no estado de Minas Gerais, Brasil, 2008 a 2017.

\begin{tabular}{|c|c|c|c|c|}
\hline \multirow[b]{2}{*}{ Condição sensível à Atenção Primária } & \multirow[b]{2}{*}{ Total } & \multicolumn{3}{|c|}{ Minas Gerais } \\
\hline & & $\%$ total & $\%$ Masc & $\%$ Fem \\
\hline 1. Doenças preveníveis por imunização & 21.108 & 0,84 & 67,42 & 32,58 \\
\hline 2. Gastroenterites Infecciosas e complicações & 286.131 & 11,41 & 49,16 & 50,84 \\
\hline 3. Anemia & 14.021 & 0,56 & 42,60 & 57,40 \\
\hline 4. Deficiências nutricionais & 87.640 & 3,50 & 56,71 & 43,29 \\
\hline 5. Infecções de ouvido, nariz e garganta & 24.079 & 0,96 & 51,86 & 48,14 \\
\hline 6. Pneumonias bacterianas & 220.605 & 8,80 & 52,06 & 47,94 \\
\hline 7. Asma & 113.218 & 4,52 & 50,79 & 49,21 \\
\hline 8. Doenças pulmonares & 211.963 & 8,46 & 54,94 & 45,06 \\
\hline 9. Hipertensão & 65.134 & 2,60 & 40,22 & 59,78 \\
\hline 10. Angina & 171.977 & 6,86 & 57,09 & 42,91 \\
\hline 11. Insuficiência cardíaca & 374.444 & 14,94 & 49,04 & 50,96 \\
\hline 12. Doenças cerebrovasculares & 238.084 & 9,50 & 51,61 & 48,39 \\
\hline 13. Diabetes mellitus & 155.299 & 6,20 & 44,28 & 55,72 \\
\hline 14. Epilepsias & 59.820 & 2,39 & 59,99 & 40,01 \\
\hline 15. Infecção no rim e trato urinário & 277.191 & 11,06 & 28,78 & 71,22 \\
\hline 16. Infecção da pele e tecido subcutâneo & 85.691 & 3,42 & 53,22 & 46,78 \\
\hline 17. Doença Inflamatória órgãos pélvicos femininos & 32.090 & 1,28 & - & 100,00 \\
\hline 18. Úlcera gastrointestinal & 43.054 & 1,72 & 64,57 & 35,43 \\
\hline 19. Doenças relacionadas ao pré- natal e parto & 25.233 & 1,01 & 8,48 & 91,52 \\
\hline Total & 2.506 .782 & 100,0 & 47,98 & 52,02 \\
\hline
\end{tabular}

Fonte: Sistema de Informação Hospitalar do Sistema Único de Saúde (SIH/SUS). Dados do Departamento de Informática do Sistema Único de Saúde (DATASUS Datasus- www.datasus.gov.br). Ministério da Saúde. Brasil

ICSAP: Internações por Condições Sensíveis à Atenção Primária; Masc: sexo masculino; Fem: sexo feminino

Em relação ao sexo, destaca-se a insuficiência cardíaca $(14,94 ; 374.444)$, seguida das gastroenterites infecciosas (11,41\%; 286.131) e infecções no rim e trato urinário (11,06\%; 277.191), e, assim como no contexto geral das causas mais frequentes, as patologias supracitadas estiveram mais presentes em mulheres (Tabela $\mathrm{V}$ ).

Considerando as causas mais frequentes por faixa etária, é possível observar que, em menores de um ano, as doenças pulmonares, as gastroenterites infecciosas e as pneumonias bacterianas assumem mais de $60 \%$ (83.925) das causas de ICSAP. De 1 a 13 anos, as gastroenterites infecciosas e complicações, a asma e as pneumonias bacterianas representam mais de 70\% (217.788) das causas de ICSAP. De 14 a 39 anos, infecções no rim e trato urinário e as gastroenterites infecciosas e complicações representam mais de 80\% (116.165) das causas de ICSAP. Por fim, na população acima de 40 anos, a insuficiência cardíaca representou quase $35 \%$ (355.378) das causas de ICSAP (Tabela V).

\section{DISCUSSÃo}

O percentual de internações por condições sensíveis à atenção primária (ICSAP) nos últimos dez anos, no estado de Minas Gerais, corresponde a $21,21 \%$ do cenário geral de internações realizadas no período pelo SUS. Embora esse percentual seja significativo para o cenário de hospitalizações no estado, as ICSAP vêm diminuindo ao longo dos anos no cenário de saúde do Brasil(12). 
A redução no número de internações pode ser consequência das mudanças que vêm acontecendo na APS, pois, quando os serviços implementam novas estratégias de prevenção, controlam de maneira eficaz os pacientes com doenças crônicas e auxiliam no diagnóstico precoce das doenças ${ }^{(13)}$.

Apesar da redução nas ICSAP, os gastos com os serviços de internação ainda são significativamente altos. Esses gastos são representativos nos serviços de saúde e afetam, principalmente, os serviços na atenção básica, por isso são importantes para a elaboração de novas políticas e novas estratégias capazes de diminuir os gastos com internações evitáveis ${ }^{(12)}$.

O grupo mais sensível a internações é o sexo feminino, cuja chance aumenta para as que possuem escolaridade menor, idade acima de 60 anos e que não fazem uso constantemente dos serviços de saúde, tornando-as um grupo que merece atenção redobrada ${ }^{(14)}$.

O envelhecimento populacional atual pode ser uma das justificativas para a maior prevalência em pessoas com idade acima de 60 anos, pois isso ocasiona sobrecarga nos serviços para o público nessa faixa etária. Dessa maneira, aumenta, também, o número de ICSAP nessa idade, com doenças relacionadas, principalmente, aos sistemas cardiovascular e respiratório(15).

O número de internações na faixa etária menor de 13 anos não foi representativo na presente pesquisa, porém outros estudos revelam que crianças menores de cinco anos também estão mais vulneráveis as ICSAP. Isto ocorre não só Brasil, mas também em países como Austrália e Inglaterra ${ }^{(9,16)}$.

As reduções que vêm sendo apresentadas nos números de ICSAP é a representação da organização do sistema de saúde, porém, infelizmente, esses números não diminuem ainda mais pelo fato dos serviços ainda sentirem dificuldade para trabalhar de forma integralizada, o que impede a diminuição nas ocorrências de internação( ${ }^{(17)}$.

O Sistema Nacional Britânico, nos anos de 2001 a 2011, mostrou um aumento nas ICSAP(16). Um estudo conduzido pelo Banco Interamericano de Desenvolvimento (BID) acerca de hospitalizações evitáveis e fortalecimento da atenção primária, utilizando-se de dados secundários em seis países da América Latina (Argentina, Colômbia, Costa Rica, Equador, México e Paraguai), avaliou 39 milhões de internações. Os resultados classificaram como ICSAP $14,3 \%$ das internações, e a taxa média variou de 10,8\% (Costa Rica) a 21,6\% (Colômbia) $)^{(18)}$.

No Brasil, mesmo com a redução no número de casos, em 2010, as internações por condições sensíveis à atenção primária representaram $22,5 \%$ das internações nos serviços públicos de saúde ${ }^{(19)}$.

As diferenças observadas entre as taxas de internação nas microrregiões são devidas a serviços que são oferecidos em cada uma delas, ou seja, se a região tem cobertura da atenção básica e, mesmo assim, o número de casos de ICSAP não tem diminuído, pode-se sugerir inadequações no modelo assistencial e problemas na qualidade do cuidado ${ }^{(20)}$.

Entre as causas principais de internação estão as doenças cardiovasculares, doenças pulmonares, gastroenterites infecciosas e complicações, infecções no rim e trato urinário. Sendo as doenças do aparelho circulatório, como insuficiência cardíaca, angina e doenças cerebrovasculares, as mais prevalentes na faixa etária de maiores de 60 $\operatorname{anos}^{(21)}$.

As internações por doenças do aparelho circulatório entre os idosos brasileiros são responsáveis por quase $40 \%$ de todos os gastos com internações hospitalares, pois algumas dessas doenças demandam internações longas e assistência de alta complexidade e tecnologia. Além disso, as doenças que acometem essa faixa etária são, em grande parte, doenças crônicas, o que requer maior qualidade no acompanhamento, o que também aumenta os gastos para o SUS ${ }^{(22,23)}$.

Poucos estudos são realizados sob a perspectiva dos gastos com as ICSAP, portanto, ainda que identificada a redução do percentual do total de gastos com ISCAP ao longo dos anos nas microrregiões de saúde do Brasil, essa redução ainda é pouco significativa, evidenciando a necessidade de intensificar ações específicas ${ }^{(24)}$.

Desse modo, podemos levar em consideração que, para que o estado de Minas Gerais consiga ter gastos menores com as internações, é importante determinar onde e como deve mudar seus serviços, atuando no desenvolvimento de ações de saúde que ultrapassem o modelo curativista e construam modelos de atenção voltados para a prevenção e promoção da saúde ${ }^{(25)}$.

Para que isso aconteça, talvez seja importante que os profissionais da Atenção Primaria à Saúde (APS) sejam orientados quanto à importância da promoção da saúde, pois alguns ainda a desconhecem, o que demostra a necessidade de discussões sobre o assunto ${ }^{(26)}$. Assim, ao oferecer novos conhecimentos aos profissionais, será possível melhorar os serviços ofertados à população e diminuir gastos com hospitalizações evitáveis ${ }^{(27)}$.

Além disso, a diminuição do gasto com ICSAP poderia favorecer a capacidade de investimento do estado de Minas Gerais também a partir da melhoria na eficiência no uso do recurso financeiro, que é responsabilidade primeira da 
gestão pública. As ICSAP podem ser ocasionadas por diversas causas, sendo as mais comuns as infecções do rim e trato urinário, pneumonias bacterianas e insuficiência cardíaca, gastroenterite infecciosa e complicações, doenças crônicas, diabetes mellitus, hipertensão arterial, infecções da pele e subcutânea, e insuficiência cardíaca(13,20,28).

Vale ressaltar que muitas dessas causas podem e devem ser observadas com cuidado na atenção básica, portanto, cada região deve analisar suas condições socioeconômicas e sua rede de serviço para, assim, definir quais estratégias deverão ser realizadas ${ }^{(28)}$.

É possível observar que os dados de Minas Gerais divergem um pouco dos dados nacionais, inicialmente pelo fato de que as doenças do aparelho circulatório apresentam o maior percentual dentre as ICSAP, como também as doenças endócrinas e geniturinárias, já os resultados para as doenças do aparelho respiratório apresentam-se com discreta diferença, sendo os resultados nacionais ainda superiores ao do estado.

Dentre as regiões de Minas Gerais responsáveis pelos maiores números de ICSAP, encontrou-se a região de Jequitinhonha. Isto ocorre, pois, a APS da região não está sendo efetiva na assistência que tem sido prestada. Um estudou evidenciou a ineficiência dos serviços de promoção à saúde e ao cuidado integral, dificultando a contribuição para a ampliação e qualidade dos serviços de saúde ${ }^{(29)}$.

Inicialmente, para entender o motivo de tal resultado, buscou-se identificar a tipologia das macrorregiões, levando em consideração o desenvolvimento socioeconômico (DSE) e a oferta de serviços de saúde (OSS). Evidenciouse, através desses parâmetros, a persistência das desigualdades regionais no estado de Minas Gerais. No caso das macrorregiões Jequitinhonha e Nordeste, ambas se destacam com baixos percentuais de desenvolvimento socioeconômico e, consequentemente, menor concentração de serviços, equipamentos e recursos humanos especializados ${ }^{(30)}$.

Os parâmetros populacionais são importantes para orientar os gestores quanto ao planejamento e priorização das ações que deverão ser envolvidas, considerando o número de habitantes, fatores epidemiológicos, demográficos e os recursos humanos, tecnológicos e financeiros disponíveis ${ }^{(31,32)}$. Com base na Política Nacional de Atenção Básica (PNAB), o grau de vulnerabilidade definirá a quantidade de pessoas por equipe ${ }^{(33)}$.

Para atender às demandas específicas de cada macrorregião, os sistemas contam com a resolubilidade, que é a capacidade em resolver as relações de saúde e doença dos usuários para atendê-los de maneira adequada, independentemente do nível de atenção ${ }^{(34)}$. Um estudo de resolubilidade efetuado com base no desempenho da prestação de serviços hospitalares por nível de atenção, no ano de 2010 , revelou que a Macro Nordeste apresentou resolubilidade abaixo de $40 \%$, enquanto a Jequitinhonha apresentou resolubilidade apenas de $28 \%^{(35)}$.

Um estudo realizado com foco na resolubilidade das macrorregiões de saúde demonstrou que a maior parcela das áreas desprovidas de serviços encontra-se na porção setentrional do estado, na qual se encontram as macros Nordeste, Jequitinhonha e Noroeste. Nesse mesmo estudo, o autor afirma que os residentes da macro Jequitinhonha são atendidos em outras localidades, e a razão de procedimentos realizados por habitante $(0,00222)$ é próxima à observada na macro Nordeste $(0,00252)^{(10)}$.

É importante propor uma organização do sistema de saúde que considere, conjuntamente, a atenção às condições agudas (geralmente de curso curto, respondidas por um sistema reativo e ações episódicas) e às condições crônicas (de curso mais longo, exigentes de um sistema de resposta proativa, contínua e integrada) ${ }^{(36)}$.

Ainda, destaca-se que uma atenção primária forte, nos moldes da Estratégia de Saúde da Família, surge como uma das possibilidades para o rompimento desse distanciamento entre a análise das condições de saúde da população e a organização dos sistemas de saúde, salientando a eficiência e efetividade de sua capacidade de resposta às reais necessidades de saúde da população(36).

A PNAB de 2011 reforça a importância do fortalecimento da Estratégia Saúde da Família para o alcance de uma maior resolutividade na atenção primária, capaz de aumentar a cobertura, promovendo cuidados integrais e desenvolvendo a promoção da saúde, tornando-se a porta de entrada para o Sistema Único de Saúde, contribuem significativamente para que a APS exerça seu papel de coordenadora e ordenadora das Redes de Atenção à Saúde ${ }^{(33,37)}$.

Diante disso, o presente estudo permitiu observar a ocorrência das ICSAP no período de 2008 a 2017 e entender que, embora tal percentual esteja decrescendo, ainda representa mais de $20 \%$ do total de internações realizadas. Com relação ao impacto financeiro dessas internações no estado de Minas Gerais, esses gastos são relevantes e que poderiam ser redirecionados para a ampliação e o fortalecimento da APS.

Assim, o resultado da APS, ou a resolutividade, como está neste trabalho descrito, refere-se ao cumprimento do papel do APS por parte de todos os seus atores, em detrimento aos serviços desarticulados, que são priorizados nos municípios e que refletem nesses índices ICSAP. Em que pese a complexidade da avaliação da capacidade 
resolutiva da atenção primária à saúde e/ou da acessibilidade a seus serviços, diante dos resultados encontrados, ratifica-se a análise das ICSAP no contexto do estado de Minas Gerais como um indicador que revela aspectos essenciais da organização desse ponto do sistema. É clara a necessidade de dar continuidade aos estudos nessa temática para que sirvam de base para a melhoria dos serviços de APS e para que induzam a mudança nos modelos de atenção à saúde praticados no estado de Minas Gerais e no Brasil, e ainda para que os reflexos desses avanços possam ser mensurados na consolidação de uma política de saúde mais equânime para o usuário e para o próprio sistema, que hoje está em crise.

O estudo apresenta limitações inerentes às pesquisas com dados secundários, possíveis de subnotificações, e no que diz respeito à validade da identificação da causa básica das internações. As limitações foram reduzidas através de análises de qualidade rigorosas e buscas específicas visando a diminuição total de vieses.

\section{CONCLUSÃO}

As internações hospitalares por condições sensíveis à Atenção Primária no estado de Minas Gerais seguem o padrão nacional, porém embora as internações estejam decrescendo, ainda representa mais de $20 \%$ do total de internações realizadas. A maior prevalência de internações foi de mulheres, com idade acima de 60 anos, sendo as principais causas doenças do aparelho circulatório, doenças do aparelho respiratório, doenças endócrinas, doenças geniturinárias e doenças infecciosas e parasitárias, ocorrendo em maior parte nas Macrorregiões de Jequitinhonha e Nordeste.

\section{CONFLITOS DE INTERESSE}

Não há conflito de interesses por parte dos autores.

\section{CONTRIBUIÇÕES}

Karina Gama dos Santos Sales, Luiz Carlos de Abreu e Italla Maria Pinheiro Bezerra contribuíram com a elaboração e delineamento do estudo; a aquisição análise, interpretação dos dados; e na redação do manuscrito. José Lucas Souza Ramos contribuiu com a aquisição, análise e interpretação de dados.

\section{REFERÊNCIAS}

1. Mendes ACG, Sá DAD, Miranda GMD, Lyra TM, Tavares RAW. Assistência pública de saúde no contexto da transição demográfica brasileira: exigências atuais e futuras. Cad Saúde Pública [Internet]. 2012 [acesso em 2018 Dez 10];28(5):955-64. Disponível em: https://www.scielosp.org/scielo.php?script=sci_ arttext\&pid=S0102-311X2012000500014

2. Fausto MCR, Rizzoto MLF, Giovanella L, Seid H, Bousquat $A$, Almeida PF, et al. O futuro da Atenção Primária à Saúde no Brasil. Saúde Debate [Internet]. 2018 [acesso em 2019 Out 9];42:12-14. Disponível em: https:// www.scielosp.org/article/sdeb/2018.v42nspe1/12-14/

3. Mendonça MHM, Matta GC, Gondim R, Giovanella L. Atenção primária à saúde no Brasil: conceitos, práticas e pesquisa [Internet]. Rio de Janeiro: Editora FIOCRUZ; 2018 [acesso em 2019 Out 9]. Disponível em: https:// books.google.com.br/books?hl=pt-BR\&lr=\&id=S7ibDwAAQBAJ\&oi=fnd\&pg=PP1\&dq=Aten $\% \mathrm{C} 3 \% \mathrm{~A} 7 \% \mathrm{C} 3$ \%A3o+prim\%C3\%A1ria+\%C3\%A0+sa\%C3\%BAde+no+Brasil:+conceitos,+pr\%C3\%A1ticas+e+pesquisa \&ots=jDpBv_326h\&sig=WkedxqBfR9hWoep-jq73tG3NOvU\#v=onepage\&q=Aten\%C3\%A7\%C3\%A30\%20 prim\%C3\%A1ria\%20\%C3\%A0\%20sa\%C3\%BAde

4. Boas GDLV, Pereira DVR, Santos EK A. A reforma da política nacional de atenção básica: mais um golpe contra o SUS. Anais do Seminário da Frente Nacional Contra a Privatização da Saúde: Saúde em Tempos de Retrocessos e Retirada de Direitos [Internet]; 2017 [acesso em 2019 Out 9] Out 27-29; Maceió, AL. Maceió: UFAL, 2017. Disponível em: http://www.seer.ufal.br/index.php/anaisseminariofncps/article/viewfile/3955/2786

5. Pereira BS, Tomasi E. Instrumento de apoio à gestão regional de saúde para monitoramento de indicadores de saúde. Epidemiol Serv Saúde [Internet]. 2016 [acesso em 2019 Out 9];25:411-8. Disponível em: https:// www.scielosp.org/scielo.php?pid=S2237-96222016000200411\&script=sci_abstract 
6. Souto KMB, Sena AGN, Pereira VOM, Santos LM. Estado e políticas de equidade em saúde: democracia participativa? Saúde Debate [Internet]. 2016 [acesso em 2019 Out 9];40:49-62. Disponível em: https://www. scielosp.org/article/sdeb/2016.v40nspe/49-62/pt/

7. Boing AF, Vicenzi RB, Magajewski F, Boing AC, Moretti-Pires RO, Peres KG, et al. Redução das internações por condições sensíveis à atenção primária no Brasil entre 1998-2009. Rev Saúde Pública [Internet]. 2012 [acesso em 2018 Dez 10];46(2):359-66. Disponível em: https://www.scielosp.org/scielo.php?pid=S0034$89102012000200019 \&$ script=sci_abstract

8. Pereira FJR, Silva CC, Lima EA Neto. Perfil das Internações por condições sensíveis à Atenção Primária subsidiando ações de saúde nas regiões brasileiras. Saúde Debate [Internet]. 2015 [acesso em 2018 Dez 10];40(107):1008-17. Disponível em: https://www.scielosp.org/scielo.php?pid=S0103$11042015000401008 \&$ script=sci_arttext\&tIng=pt

9. Nedel FB, Facchini LA, Martin M, Navarro A. Características da atenção básica associadas ao risco de internar por condições sensíveis à atenção primária: revisão sistemática da literatura. Epidemiol Serv Saúde [Internet]. 2010 [acesso em 2018 Dez 12];19(1):61-75. Disponível em: http://scielo.iec.gov.br/scielo. php?pid=S1679-49742010000100008\&script=sci_abstract

10. Malachias I, Leles FAG, Pinto MAS. Plano Diretor de Regionalização da Saúde de Minas Gerais [Internet]. Belo Horizonte: Secretaria de Estado de Saúde de Minas Gerais; 2011 [acesso em 2018 Dez 12]. Disponível em: https://cotec.fadenor.com.br/assets/documentos/350/anexos/PDRMG_-_Plano_Diretor_de_ Regionaliza\%C3\%A7\%C3\%A3o_da_Sa\%C3\%BAde.pdf

11. Ministério da Saúde (BR). Portaria № 221, de 17 de Abril de 2008 [Internet]. Brasília: Ministério da Saúde; [2008] [acesso em 2018 Dez 12]. Disponível em: http://bvsms.saude.gov.br/bvs/saudelegis/sas/2008/ prt0221_17_04_2008.html

12. Morimoto T, Costa JSD. Internações por condições sensíveis à atenção primária, gastos com saúde e Estratégia Saúde da Família: uma análise de tendência. Ciênc Saúde Colet [Internet]. 2017 [acesso em 2019 Out 10];22:891-900. Disponível em: https://www.scielosp.org/scielo.php?pid=S1413$81232017000300891 \&$ script=sci_arttext

13. Santos FC, Bordin R. Internações por condições sensíveis à atenção básica: uma revisão, 2005-2014. Gest Saúde Rio Gd do Sul: Casos Anal Prat [Internet]. 2017 [acesso em 2019 Out 10];2:191-208. Disponível em: https://www.lume.ufrgs.br/bitstream/handle/10183/159686/001022725.pdf?sequence=1

14. Pitilin ÉB, Gutubir D, Fernandes CAM, Pelloso SM. Internações sensíveis à atenção primária específicas de mulheres. Ciênc Saúde Colet [Internet]. 2015 [acesso em 2019 Out 10];20:441-448. Disponível em: https:// www.scielosp.org/scielo.php?pid=S1413-81232015000200441\&script=sci_arttext\&tlng=es

15. Rodrigues MM, Alvarez AM, Rauch KC. Tendência das internações e da mortalidade de idosos por condições sensíveis à atenção primária. Rev Bras Epidemiol [Internet]. 2019 [acesso em 2019 Out 10];22:190010. Disponível em: https://www.scielosp.org/article/rbepid/2019.v22/e190010/pt/

16. Laberge M, Wodchis WP, Barnsley J, Laporte A. Hospitalizations for ambulatory care sensitive conditions across primary care models in Ontario, Canada. Soc Sci Med [Internet]. 2017 [acesso em 2019 Jul 15];181:24-33. Disponível em: https://www.sciencedirect.com/science/article/abs/pii/S027795361730196X

17. Rehem TCMS, Emiko Egry Y, Ciosak SI, Santos WS. Quais aspectos contribuem para a ocorrência de internações por condições sensíveis à atenção primária? Rev Bras Promoç Saúde [Internet]. 2017 [acesso em 2019 Out 10];29:138-47. Disponivel em: https://periodicos.unifor.br/RBPS/article/view/6415/5222

18. Guanais F, Gómez-Suárez R, Pinzón L. Series of avoidable hospitalizations and strengthening primary health care: primary care effectiveness and the extent of avoidable hospitalizations in Latin America and the Caribbean [Internet]. Washington, DC: Inter-American Development Bank; 2012 [acesso em 2019 Ago 2]. Disponível em: https://publications.iadb.org/en/publication/11805/series-avoidable-hospitalizations-andstrengthening-primary-health-care-primary_

19. Viacava F, Ugá MAD, Porto S, Laguardia J, Moreira RS. Avaliação de desempenho de sistemas de saúde: um modelo de análise. Ciênc Saúde Colet [Internet]. 2012 [acesso em 2019 Ago 2];17:921-934. Disponível em: https://www.scielosp.org/scielo.php?pid=S1413-81232012000400014\&script=sci_arttext\&tlng=en 
20. Magalhães ALA, Morais NOL. Desigualdades intraurbanas de taxas de internações por condições sensíveis à atenção primária na região central do Brasil. Ciênc Saúde Colet [Internet]. 2017 [acesso em 2019 Out 11];22:2049-62. Disponível em: https://www.scielosp.org/scielo.php?pid=S1413$81232017000602049 \&$ script=sci_arttext

21. Marques AP, Montilla DER, Almeida WS, Andrade CLT. Internação de Idosos por condições sensíveis à atenção primária à saúde. Rev Saúde Pública [Internet]. 2014 [acesso em 2019 Out 11];48(5):817-26. Disponível em: http://www.periodicos.usp.br/rsp/article/view/86923/89893

22. Peixoto SV, Giatti L, Alfradique ME, Lima-Costa MF. Custo das internações hospitalares entre idosos brasileiros no âmbito do Sistema Único de Saúde. Epidemiol Serv Saude [Internet]. 2004 [acesso em 2019 Ago 2];13(4):239-46. Disponível em: http://scielo.iec.gov.br/pdf/ess/v13n4/v13n4a06.pdf

23. Kernkamp CL, Costa CKF, Massuda EM, Silva ES, Yamaguchi MU, Bernuci MP. Perfil de morbidade e gastos hospitalares com idosos no Paraná, Brasil, entre 2008 e 2012. Cad Saúde Pública [Internet]. 2016 [acesso em 2019 Out 11];32:00044115. Disponível em: https://www.scielosp.org/scielo.php?pid=S0102311X2016000705005\&script=sci_arttext\&tlng=en

24. Macinko J, Oliveira VB, Turci MA, Guanais FC, Bonolo PF, Lima-Costa MF. The influence of Primary Care and Hospital Supply on Ambulatory Care-Sensitive Hospitalizations Among Adults in Brazil, 1999-2007. Am J Public Health Res [Internet]. 2011 [acesso em 2019 Ago 2];101(10):1963-70. Disponível em: https://ajph. aphapublications.org/doi/abs/10.2105/AJPH.2010.198887

25. Bezerra IMP, Sorpreso ICE. Concepts and movements in health promotion to guide educational practices. J Hum Growth Dev [Internet]. 2016 [acesso em 2019 Out 12];26(1):11-20. Disponível em: https://www.revistas. usp.br/jhgd/article/view/113709/112279

26. Sobral LIL, Machado LDS, Gomes SHP, Pequeno AMC, Nuto SAS, Machado MFAS. Conhecimento de profissionais da atenção básica sobre as competências de promoção da saúde. Rev Bras Promoç Saúde [Internet]. 2018 [acesso em 2019 Out 12];31(2). Disponível em: https://periodicos.unifor.br/RBPS/article/ view/6653/pdf

27. Bezerra IMP. Translational medicine and its contribution to public health. J Hum Growth Dev [Internet]. 2017 [acesso em 2019 Out 12];27(1):6-9. Disponível em: http://www.periodicos.usp.br/jhgd/article/ view/127642/130087

28. Santos BV, Lima DS, Fontes CJF. Internações por condições sensíveis à atenção primária no estado de Rondônia: estudo descritivo do período 2012-2016. Epidemiol Serv Saúde [Internet]. 2019 [acesso em 2019 Out 12];28:2017497. Disponível em: https://www.scielosp.org/article/ress/2019.v28n1/e2017497/

29. Paula FA, Silva CCR, Santos DF, Martins-Filho OA, Andrade RA. Avaliação da atenção à saúde do adulto em um município-polo do Vale do Jequitinhonha (MG). Saúde Debate [Internet]. 2015 [acesso em 2019 Out 12];39:802-14. Disponível em: https://www.scielosp.org/scielo.php?pid=S0103$11042015000300802 \&$ script=sci_arttext\&tIng=en

30. Silva LFE. Internações por Condições Sensíveis à Atenção Primária em Minas Gerais: Análise da prevalência e dos gastos nas macrorregiões de saúde [dissertação]. Juiz de Fora: Universidade Federal de Juiz de Fora; 2017.

31. Ministério da Saúde (BR). Portaria n. ${ }^{\circ}$ 1101/GM, 12 de junho de 2002. Brasília: Ministério da Saúde; [2002].

32. Ministério da Saúde (BR), Secretaria de Políticas De Saúde, Departamento de Atenção Básica. Parâmetro para programação das ações básicas de saúde. Brasília: Ministério da Saúde; 2001.

33. Brasil. Ministério da Saúde. Portaria $n^{\circ} 2.488$, de 21 de outubro de 2011. Aprova a Política Nacional de Atenção Básica, estabelecendo a revisão de diretrizes e normas para a organização da Atenção Básica, para a Estratégia Saúde da Família (ESF) e o Programa de Agentes Comunitários de Saúde (Pacs). Diário Oficial da República Federativa do Brasil. Brasília, DF, 22 Out. 2011.

34. Palmeira SS, Pereira TM, Almeida TL, Sousa AR, Alencar DC. Resolubilidade dos serviços ofertados na estratégia saúde da família: discurso de homens. Saúde Redes [Internet]. 2018 [acesso em 2019 Out 12];4(4):105-17. Disponível em: http://revista.redeunida.org.br/ojs/index.php/rede-unida/article/view/1836/pdf 
35. Malachias I, Marra A, Castro GB, Pinto MAS, Siqueira M, Azevedo J. A resolubilidade e os vazios da assistência hospitalar micro e macrorregional do SUS/MG em 2010 e a evolução-2003/2010 [Internet]. Belo Horizonte: Secretaria de Estado de Saúde de Minas Gerais; 2011 [acesso em 2019 Ago 2]. Disponível em: http://www.saude.mg.gov.br/images/documentos/2A\%20Resolubilidade $\% 20 \mathrm{e} \% 20$ os $\% 20$ vazios $\% 20$ da\%20 Assistenc.pdf

36. Mendes EV. As redes de atenção à saúde. Cienc Saúde Colet [Internet]. 2010 [acesso em 2019 Ago 2];15(5):2297-305. Disponível em: https://www.scielosp.org/scielo.php?pid=S1413$81232010000500005 \&$ script=sci_arttext

37. Morosini MVGC, Fonseca AF, Lima LD. Política Nacional de Atenção Básica 2017: retrocessos e riscos para o Sistema Único de Saúde. Saúde Debate [Internet]. 2018 [acesso em 2019 Out 12];42:11-24. Disponível em: https://www.scielosp.org/article/sdeb/2018.v42n116/11-24/pt/

\section{Endereço para correspondência:}

Karina Gama dos Santos Sales

Escola Superior de Ciências da Santa Casa de Misericórdia de Vitória - EMESCAM

Av. Nossa Sra. da Penha, 2190

Bairro: Bela Vista

CEP: 29027-502 - Vitória - ES - Brasil

E-mail: karina.gamadossantos@gmail.com

Como citar: Sales KGS, Abreu LC, Ramos JLS, Bezerra IMP. Internações hospitalares por condições sensíveis à atenção primária à saúde. Rev Bras Promoç Saúde. 2019;32:9664. 